\title{
Hospital prevalence and major causes of low vision in a tertiary care centre in South India: A Hospital record based study
}

\author{
Shimna C $\mathbf{P}^{1, *}$, Jasmine Mary Jacob ${ }^{2}$, Pramod Thomas ${ }^{3}$ \\ ${ }^{1}$ Assistant Professor, ${ }^{2}$ Professor, Dept. of Ophthalmology, Dr Somervell Memorial CSI Medial College, Karakonam, Kerala, \\ ${ }^{3}$ Associate Professor, Dept. of Community Medicine, Believers Church Medical College, Thiruvalla, Kerala, India
}

*Corresponding Author:

Email: shimna1978@gmail.com

\begin{abstract}
Aims: To determine the hospital prevalence as well as the low vision causes among patients coming to a tertiary care centre. Settings and Design: Retrospective study was conducted by review of files of patients who presented to the Ophthalmology OPD from September 2013 to February 2014.

Material and Methods: Records of 4989 patients in the 20-60 years age group who presented to the Ophthalmology OPD were collected and data obtained. Patients with a visual acuity of $<6 / 18(20 / 60)$ to $\geq 3 / 60(20 / 400)$ in the best eye were included in the low vision group according to the WHO definition. ${ }^{3}$

Results: There were 102 patients with low vision who attended the OPD during the study period. There were $36(35 \%)$ male and $66(65 \%)$ female patients among the low vision patients. The low vision causes were retinal diseases (72\%), amblyopia (15\%), corneal opacity $(9 \%)$ and glaucoma $(4 \%)$.

Conclusion: Hospital prevalence of low vision was estimated to be $2.19 \%$ (SE-1.43). Among these patients, the causes of low vision were detected to be Retinal diseases like Diabetic Retinopathy, Hypertensive Retinopathy and pathological myopia, amblyopia, corneal opacity and glaucoma.
\end{abstract}

Keywords: Low vision, Diabetic Retinopathy (DR), Age Related Macular Degeneration (ARMD), Hypertensive Retinopathy (HTR), Retinitis Pigmentosa (RP).

\section{Introduction}

It has been estimated that about 284 million people are visually impaired worldwide, in which 54.5 million have low vision in India. ${ }^{1}$ According to the global data of visual impairment by WHO in 2010, low vision in India was estimated to be around $22.2 \%$ (54.5 million) and in Europe around $10.4 \%$ (25.5 million). ${ }^{1}$ The prevalence of low vision is $3.65 \%$ globally, but it is estimated to be $1.05 \%$ in India. ${ }^{2}$ Globally, the major causes of low vision were recorded as retinal pathologies like Diabetic retinopathy (DR), Age related macular degeneration (ARMD), glaucoma and amblyopia.

Worldwide, low vision is a significant public health problem which can affect the quality of life. An individual with low vision may find it difficult in accomplishing daily activities like reading, writing, shopping, watching television, or even recognizing faces. Even after treatment and/or standard refractive correction, a person with low vision has impairment of visual functioning. Low vision patients have a visual acuity of less than $<6 / 18(20 / 60)$ to $\geq 3 / 60(20 / 400)$ in the better eye and /or a visual field of less than 20 degrees from the point of fixation. ${ }^{3}$ Appropriate intervention can help these patients to use vision for the planning and/or execution of a task.

In Southern India, a population based cross sectional study was done among 10,293 persons from 94 clusters of all ages. In this study, the prevalence of low vision was estimated to be $1.05 \%$. Major causes of low vision in this study were retinal diseases, amblyopia, optic atrophy, glaucoma and corneal diseases. ${ }^{4}$ Another study done among the urban South Indian population of 3850 subjects, known as the Chennai Glaucoma study, the prevalence of low vision was $2.9 \%$ (95\% CI 2.4-2.4\%) and most common causes of low vision were refractive errors and cataract. ${ }^{5}$

\section{Materials and Methods}

A retrospective study was done in the Department of Ophthalmology in a tertiary care centre in South Kerala. Medical records were obtained from the medical records department with written permission from the concerned authority. Approval for the study protocol was obtained from Institutional review board (IRB) and Ethical Committee which constituted members outside the institution as per ICMR guidelines required for any study conducted in the institution.

Records of patients of 20-60 years age group who presented to the Ophthalmology OPD from September 2013 to February 2014 were included in the study. Information on age, sex, Snellen's best corrected visual acuity, slit lamp examination findings, fundus examination findings and diagnosis of 4989 patients were obtained from hospital records. Patients who had a visual acuity of $<6 / 18(20 / 60)$ to $\geq 3 / 60(20 / 400)$ in the best eye even after refractive error correction, cataract surgery or treatment of retinal pathologies, were included in the low vision group. Patients who had best corrected visual acuity of $\geq 6 / 18 \quad(20 / 60)$, after correction of refractive error or post cataract surgery were excluded from the study because their vision improved beyond 6/18 (20/60) after the intervention and could no longer be included under the low vision 
group according to the WHO definition. ${ }^{3}$ Data entered into an excel sheet and the data analysis was done using Statistical software R and Microsoft Excel. A univariate analysis of variables such as age, sex as well as causes of low vision was done in the study.

\section{Results}

The data collected from the files of 4989 patients were analysed. Out of the 4989 files analysed, 102 patients were found to have low vision. The hospital prevalence of low vision in this study was estimated to be $2.19 \%$ (SE-1.43) among the study population.

Among the 102 files analysed, $36(35 \%)$ were male patients with mean age of 48.2 years (SD - 10.2) and 66 $(65 \%)$ were female patients with mean age of 49.5 years (SD - 9.7) [Fig. 1]. We divided the age group into 2 category, of which one included subjects between 20 to 50 years age group and the other included 51 to 60 years age group. Among the 20-50 years age group, there were $18(18 \%)$ male and $30(29 \%)$ female patients whereas there were $18(18 \%)$ male and $36(35 \%)$ female patients in the age group of 51-60 years [Fig. 2].

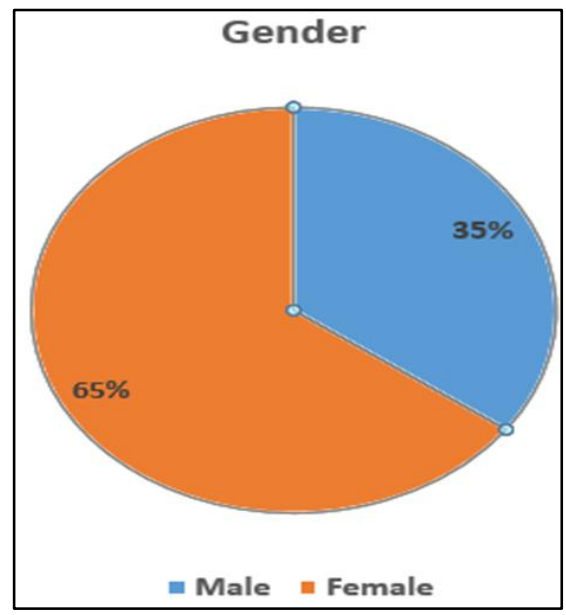

Fig. 1: Gender distribution of participants with low vision

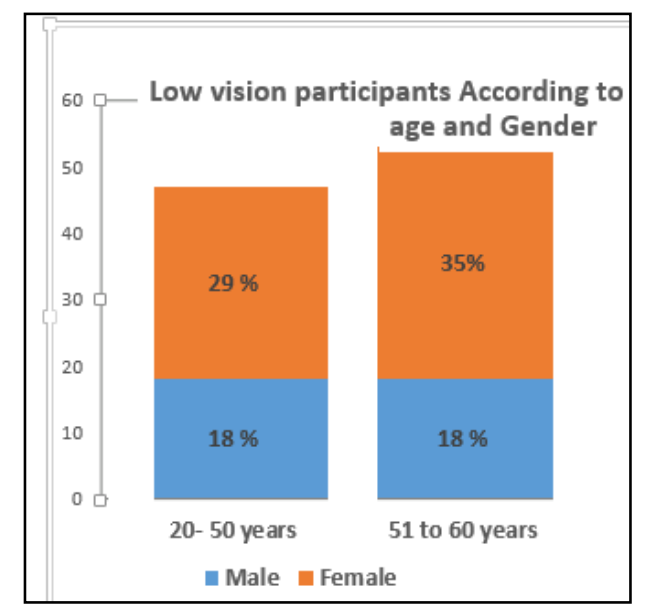

Fig. 2: Low vision causes participants according to age and gender

Causes of low vision were detected to be retinal diseases, corneal opacity, advanced glaucoma with visual fields less than $20^{\circ}$ and amblyopia. Diabetic Retinopathy (DR) cases who did not regain vision $\geq$ $6 / 18$ even after laser treatment or vitrectomy, Hypertensive retinopathy (HTR), Retinitis Pigmentosa (RP), Age Related Macular Degeneration (ARMD), Pathological Myopia, Idiopathic macular hole with failed ILM peeling surgeries and Retinal Detachment (RD) post failed surgeries were detected among the retinal disease patients with low vision.

Among the 102 low vision patients, 74 (72\%) patients had retinal diseases. Out of the 74 patients, 36 (48.64\%) patients had DR, $23(31.08 \%)$ patients had pathological myopia, $4(5.41 \%)$ patients had RP, 4 $(5.41 \%)$ patients had HTR, $3(4.05 \%)$ patients had ARMD, 2 (2.7\%) patients had Idiopathic macular hole and $2(2.7 \%)$ patients had Retinal Detachment. 15 (15\%) patients out of the 102 files analysed had amblyopia, 9 (9\%) patients had corneal opacity, and 4 (4\%) patients had glaucoma [Fig. 3].

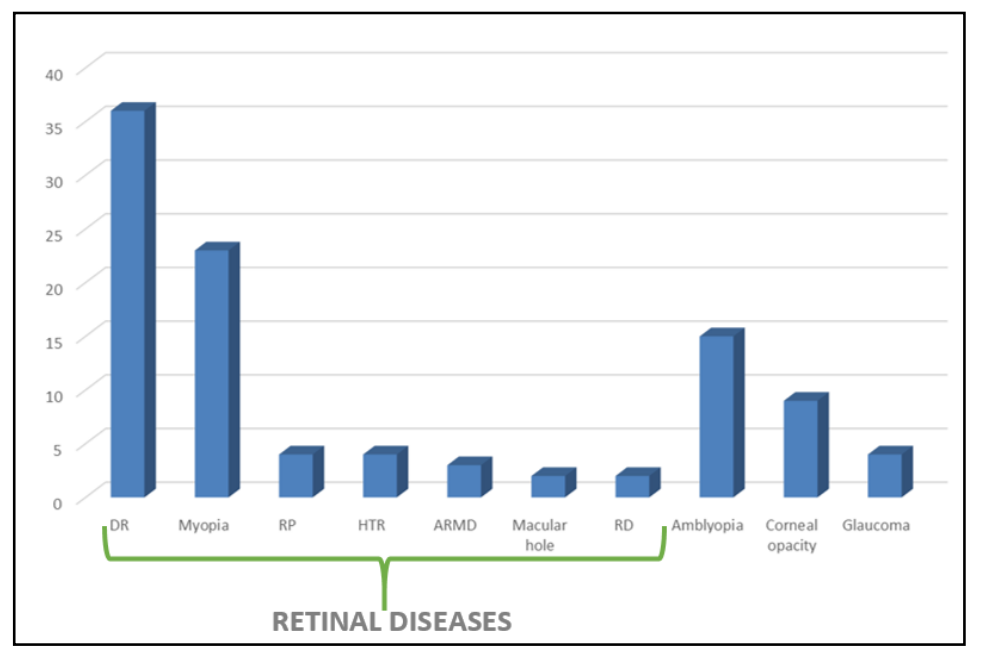

Fig. 3: Low vision causes among the study group 
Retinal diseases were more among the female patients compared to their male counterparts. Among the 74 patients with retinal diseases, $50(49.02 \%)$ were female patients as compared to males which was only $24(23.53 \%)$. Corneal opacity was found to be more among the males compared to females. Out of 9 corneal opacity cases, $7(6.86 \%)$ patients were male compared to females which was only $2(1.96 \%)$.

$12(11.8 \%)$ out of 15 patients who had amblyopia were females compared to males which was only 3 (2.9\%). Only 4 patients among 102 patients had glaucoma and all 4 (3.9\%) were female patients. There were no male patients with glaucoma among the files analysed.

We also analysed the causes of low vision according to age group of the patients. $13(12.7 \%)$ males and $21(20.58 \%)$ females were detected to have retinal diseases in the age group of 20-50 years whereas $15(14.7 \%)$ males and $25(24.5 \%)$ females were among the age group of 51-60 years. The number of females with retinal diseases were more in both the groups, compared to males.

There were $2(1.96 \%)$ males and $7(6.86 \%)$ females among 20-50 years age group and $2(1.96 \%)$ male and 4 (3.9\%) females in 51-60 years age group who had amblyopia. Out of the patients with corneal opacity, there were $4(3.9 \%)$ males and $1(0.98 \%)$ female in the $20-50$ years age group and $3(2.9 \%)$ males and 1 $(0.98 \%)$ female in the 51-60 years age group. There were only female patients who had glaucoma in both the age groups, $1(0.98 \%)$ among $20-50$ years and 3 (2.9\%) among 51-60 years [Table 1].

Table 1: Gender distribution of causes of low vision:

\begin{tabular}{|l|c|c|c|c|c|}
\hline Causes of low vision & \multirow{2}{*}{ Total Patients } & \multicolumn{2}{|c|}{ Males } & \multicolumn{2}{c|}{ Females } \\
\cline { 3 - 6 } & & $\mathbf{2 0 - 5 0}$ years & $\mathbf{5 1 - 6 0}$ years & $\mathbf{2 0 - 5 0}$ years & $\mathbf{5 1 - 6 0}$ years \\
\hline Retinal diseases & $74(72 \%)$ & $13(12.7 \%)$ & $15(14.7 \%)$ & $2120.58 \%)$ & $25(24.5 \%)$ \\
\hline Corneal opacity & $9(9 \%)$ & $4(3.9 \%)$ & $3(2.9 \%)$ & $1(0.98 \%)$ & $1(0.98 \%)$ \\
\hline Amblyopia & $15(15 \%)$ & $2(1.96 \%)$ & $2(1.96 \%)$ & $7(6.86 \%)$ & $4(3.9 \%)$ \\
\hline Glaucoma & $4(4 \%)$ & $0(0 \%)$ & $0(0 \%)$ & $1(0.98 \%)$ & $3(2.9 \%)$ \\
\hline
\end{tabular}

\section{Discussion}

In this retrospective hospital record based study, 4989 files were collected out of which 102 patients were found to have low vision and the estimated proportion of low vision was $2.19 \%$ (SE-1.43). There were no other hospital record based retrospective studies in low vision available in the literature though there were a few population based study which analysed the low vision prevalence and its causes.

In literature, only 2 prospective population based cross sectional studies were found to be conducted in India. It was found that in one cross sectional study done in an urban population in South India, the low vision prevalence was $2.9 \%{ }^{5}$ which was consistent with our study. But in the second population based study done in Southern India, among 10,293 persons from 94 clusters of all ages, prevalence of low vision was found to be $1.05 \%$ which was less than the prevalence in our study. The difference in prevalence rate may be due to the difference in study population, in which ours was hospital record based and theirs was community based.

In our study population, major low vision causes were found to be retinal diseases, amblyopia, corneal opacity and glaucoma. Major retinal diseases were DR and pathological myopia and the less common being RP, HTR, ARMD, Idiopathic macular hole and RD. In Andhra Pradesh Eye disease study, main low vision causes were retinal diseases and amblyopia ${ }^{4}$ which was similar to our study whereas in Chennai Glaucoma study, refractive errors were the main cause of low vision, which was not consistent with our study, as we did not include those patients whose best corrected visual acuity improved with refractive error correction and cataract surgery.

There are several other international studies found in literature to find out the prevalence as well as the low vision causes. In a study conducted among the Japanese population, the overall prevalence of low vision was found to be $0.39 \%(95 \% \mathrm{CI}){ }^{6}{ }^{6}$ The study subjects were 3870 Japanese adults, in whom the leading causes of low vision were cataract and glaucoma. ${ }^{6}$ At Singapore, a study was conducted, in which the low vision prevalence was $1.0 \%^{7}$ and refractive error and cataract were the main low vision causes among the subjects. ${ }^{7} \mathrm{~A}$ retrospective study conducted among the Canadian population detected that the low vision prevalence was $2.36 \%$ among the age group of $65-74$ years $^{8}$ and they found Visual pathway disease and ARMD as the main low vision causes, DR and glaucoma being less common. ${ }^{8}$

Copenhagen city eye study was another cross sectional study done among the Scandinavian population in which they found that the rate of prevalence of low vision was $0.66 \%$ and the study found that myopia-related retinal disorders, DR, optic neuropathy, and retinitis pigmentosa (RP) were the most common low vision causes among the 20 to 64 years age group. ${ }^{9,10}$ Eye diseases prevalence research group detected the low vision prevalence among adults in United States was $1.98 \%$ and the leading low vision causes among whites was ARMD, while cataract and glaucoma were the most common among blacks. ${ }^{11}$

The Rotterdam study found the low vision prevalence of $0.1 \%$ among those who were 75 years 
and less. Myopic degeneration and optic neuropathy were the most important low vision causes in persons who were less than 75 years whereas ARMD was the major cause for persons who were 75 years or more. ${ }^{12}$ A study conducted among Chinese population residing in Taiwan known as the Shihpai eye study found the low vision prevalence was $2.94 \%$ (95\% CI). Majority of low vision causes in the study group was myopic macular degeneration and ARMD. ${ }^{13}$

The limitation of our study was that it was a retrospective study. Another drawback was that the sample size was very small compared to other studies. A significant number of those with low vision are much older than 60 years of age with AMD, but our study included only those among 20-60 years of age. This is another major limitation of the study.

\section{Conclusion}

In our study, we found that the Hospital prevalence of low vision was estimated to be $2.19 \%$ (SE-1.43). Retinal diseases like Diabetic Retinopathy and pathological myopia, amblyopia, corneal opacity and glaucoma were the low vision causes among these patients.

\section{References}

1. Orbis: Eliminating blindness in India. January 20, 2010.http://www.orbis.org/ Default.aspx?cid=5713=1.

2. Eye care in India. A situation analysis. Ch 3: 39. Sight savers international, India. 2007.

3. WHO Global data on visual impairments 2010.

4. Rakhi Dandona, Lalit Dandona, Marmamula Srinivas, Pyda Giridhar, Rishita Nutheti, Gullapalli N Rao. Planning low vision services in India: A population-based perspective. Ophthalmology Oct 2002; 109: 10:18711878. doi.org/10.1016/S0161-6420 (02)01183-1.

5. Lingam Vijaya, Ronnie George, Rashima Asokan, Lokapavani Velumuri, Sathyamangalam Ve Ramesh. Prevalence and causes of low vision and blindness in an urban population: The Chennai Glaucoma Study. IJO 2014 April; 62(4):477-481. doi:10.4103/0301-4738; 111186.

6. Aiko Iwase, Makoto Araie et al. Prevalence and causes of low vision and blindness in a Japanese Adult population: The Tajimi Study. Ophthalmology. August 2006;113:8:1354-1362.

7. Tien Yin Wong, Elaine W. Chong et al. Prevalence and Causes of Low Vision and Blindness in an Urban Malay Population. The Singapore Malay Eye Study. Arch Ophthalmol. 2008; 126(8):1091-1099. doi:10.1001/archopht.126.8.1091.

8. DAL Maberly, H Hollands et al. The prevalence of low vision and blindness in Canada. Eye. 2006. 30:341-346.

9. Buch H, Vinding T, la Cour M, Appleyard M, Jensen GB, Nielsen NV. Prevalence and causes of visual impairment and blindness among 9980 Scandinavian adults. Ophthalmology 2004;111:53-61.

10. Buch H, Troels V, Nielsen NV. Prevalence and causes of visual impairment according to World Health Organization and United States criteria in an aged, urban Scandinavian population: The Copenhagen City Eye Study. Ophthalmology 2001;108:2347-2357.

11. The eye diseases prevalence research group. Causes and Prevalence of Visual Impairment among Adults in the
United States. Arch Ophthalmol 2004; 122(4): 477-485. Doi.10.1001/archopht.122.4.477.

12. Klaver CC, Wolfs RC, Vingerling JR, Hofman A, de Jong PT. Age-specific prevalence and causes of blindness and visual impairment in an older population: the Rotterdam Study. Arch Ophthalmol 1998;116:653-658.

13. Wen-Ming Hsu, Ching-Yu Cheng, MD, Jorn-Hon Liu, $\mathrm{Su}$-Ying Tsai, Pesus Chou. Prevalence and causes of visual impairment in an elderly Chinese population in Taiwan: The Shihpai Eye Study Presented in part at: XXIXth International Congress of Ophthalmology, April, 2002; Sydney. doi:10.1016/j.ophtha.2003.05.011. 